\title{
Impact of Caspase Activation in Human Spermatozoa
}

\author{
SONJA GRUNEWALD, ${ }^{1}$ RAKESH SHARMA, ${ }^{2}$ UWE PAASCH,${ }^{1}$ \\ HANS-JUERGEN GLANDER, ${ }^{1}$ AND ASHOK AGARWAL ${ }^{2 *}$ \\ ${ }^{1}$ Department of Dermatology / Andrology Unit, University of Leipzig, 04103 Leipzig, Germany \\ ${ }^{2}$ Center for Reproductive Medicine, Glickman Urological and Kidney Institute, Cleveland Clinic Foundation, Cleveland, Ohio 44195
}

\begin{abstract}
KEYWORDS caspase assays; sperm cryopreservation; sperm maturation; sperm separation
\end{abstract}
\begin{abstract}
Caspases are central components in the apoptosis signaling cascade. The family of cysteine proteases transduces and enhances the apoptosis signal, and activation of effector caspases results in controlled cellular degradation. Although initially the presence of caspases in spermatozoa was controversially discussed in recent years, many studies demonstrated their activation in male germ cells. Activated apoptosis signaling results in decreased fertilizing capacity of the sperm. This review presents the current knowledge on the role of caspases in human sperm. Techniques of caspase monitoring are highlighted. With regard to the high impact of caspases on the sperm fertilizing potential, physiological and pathological settings of caspase activation and inactivation are discussed. Finally, the effects of depletion of caspase-positive sperm are shown with various standard and molecular sperm preparation methods. Microsc. Res. Tech. 00:000-000, 2009. ๑ 2009 Wiley-Liss, Inc.
\end{abstract}

\section{INTRODUCTION}

Male infertility is the sole or contributing factor in almost half of the couples failing to conceive (Sharlip et al., 2002). Semen analysis including the assessment of sperm concentration, motility, and percentage normal forms is the standard procedure for evaluating the male fertility potential (World Health Organization, 1999). Although the conventional analysis gives considerable information, it does not provide information about impaired subcellular processes in human sperm, and defined pathophysiological diagnosis of male infertility is often missed. In recent years, many studies have investigated the presence and significance of programmed cell death (apoptosis) in spermatozoa, which may be partially responsible for the low fertilization and implantation rates seen with assisted reproductive techniques (Oehninger et al., 2003). Caspases (CP, cysteinyl-aspartate-specific proteases) are the main transducers and effectors of the apoptosis signal leading to programmed cell death. They comprise a family of cysteine proteases that cleave proteins after aspartic acid residues. In somatic cells, their activation leads to characteristic morphological changes of the cell such as shrinkage, chromatin condensation, DNA fragmentation, and plasma membrane blebbing.

The objective of this review is to present the current knowledge on presence and functional relevance of caspase activation in human sperm. In addition, we review various techniques of caspase detection in human sperm. The potential impact of caspase activation on sperm fertilizing capacity is highlighted and standard and molecular-based sperm preparation techniques are examined with regard to their potential to deplete caspase-positive sperm.

\section{THE CASPASE SYSTEM}

Caspases were first described in the nematode C. elegans. The cell death abnormal protein 3 (ced-3) gene was demonstrated to encode a cysteine protease similar to the human interleukin-1b-converting enzyme which was later renamed to caspase-1 (Thornberry et al., 1992). To date, 14 caspases have been identified. They can be classified on the basis of their known major functions into two subfamilies, pro-apoptotic and proinflammatory subfamilies. Caspase-2, -3, -6, -7, -8, -9, -10 are known to be predominantly involved in mediating apoptosis signal transduction, whereas pro-inflammatory caspases (caspase-1, -4, -5, -11, -12) regulate cytokine maturation during inflammation. However, the activation of "pro-inflammatory" caspases can induce apoptosis and pro-apoptotic caspases can be involved in inflammation-related signaling. Moreover, caspases also play roles in other cellular processes not fitting in these categories (Kumar, 2004), among them cellular proliferation, differentiation and migration. Although the role of caspases in these processes is not fully investigated, studies proved, e.g., the essential role of caspase- 8 activation for proliferation of immune cells ( $\mathrm{T}, \mathrm{B}$ and natural killer cells). Caspases are involved in the terminal differentiation of a variety of cell types, including enucleation processes such as lens cell differentiation, erythrocyte and platelet formation and the terminal differentiation of keratinocytes. Transient activation of caspase-3 mediates differentiation of long-lived cell types, such as skeletal muscle, osteoblasts and neurons. Cell migration has been shown to be severely hampered in caspase-8-deficient mouse embryonic fibroblasts. How caspase activity is restrained and guided to influence differentiation without induc-

\footnotetext{
*Correspondence to: Ashok Agarwal, Ph.D., H.C.L.D., Professor and Director, Center for Reproductive Medicine, Glickman Urological and Kidney Institute and Obstetrics and Gynecology and Women's Health Institute, Cleveland Clinic, 9500 Euclid Avenue, Desk A19.1, Cleveland, Ohio 44195.

E-mail: agarwaa@ccf.org

Received 15 December 2008; accepted in revised form 1 April 2009

DOI 10.1002/jemt.20732

Published online in Wiley InterScience (www.interscience.wiley.com).
} 


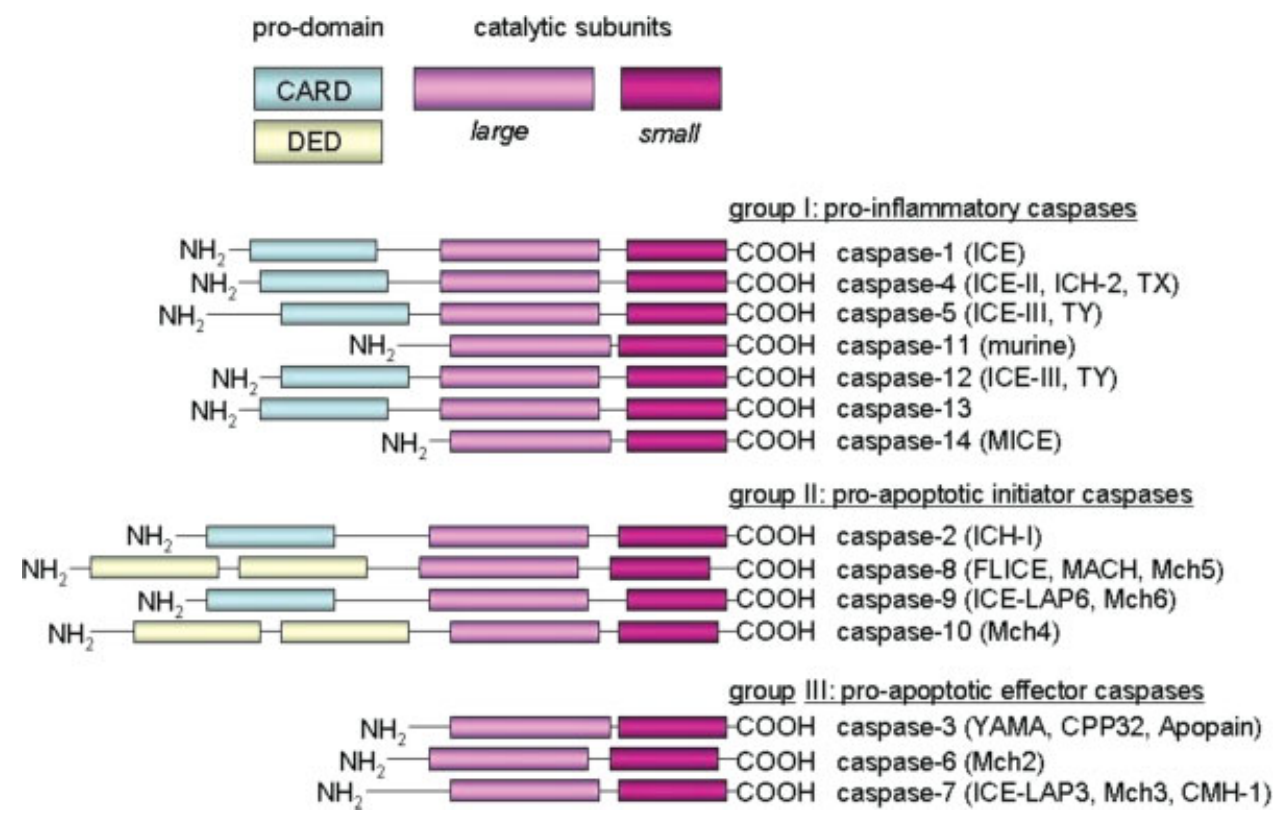

Fig. 1. Caspase classification based on structure and function of the proteases. [Color figure can be viewed in the online issue, which is available at www.interscience.wiley.com.]

ing cell death awaits future studies ( $\mathrm{Li}$ and Yuan, 2008).

Alternatively, caspases can be classified according to the lengths of their prodomains, which also corresponds to their positions in the apoptosis signaling cascade. Initiator caspases (caspase-1, -2, -4, -5, -8, -9, -10, $-11,-12$ ) possess long prodomains that contain one of the two characteristic protein-protein interaction motifs: the death effector domain (DED) or the caspase recruitment domain (CARD). They interact with the upstream adapter molecules and activate effector caspases. Effector caspases (caspase-3, -6, -7) contain short prodomains and execute the apoptosis signal by cleaving a variety of cellular substrates (Cryns and Yuan, 1998). A complete overview on caspase classification is given in Figure 1.

Like most proteases, caspases are synthesized as inactive zymogens containing a prodomain, a $20 \mathrm{kD}$ large subunit and a $10 \mathrm{kD}$ small subunit. Activation of the zymogens by proteolytic cleavages separates the large and small subunits and removes the prodomain, followed by association of the large and small subunits and formation of a heterodimer (Thornberry and Lazebnik, 1998). The catalytic center is located in the $20 \mathrm{kD}$ subunit. Caspases recognize at least four contiguous amino acids in their substrates and cleave after the C-terminal residue, usually an aspartic residue (Fuentes-Prior and Salvesen, 2004).

Although the majority of caspases are situated within the cytoplasm, some members can be found in association to the mitochondria (CP-2, -3 and -9) or at the Golgi apparatus (CP-12) (Nicholson, 1999). The most important "initiator" caspases are caspase-8 for receptor-mediated (type I) apoptosis, caspase-9 for mitochondria-related (type II) apoptosis, and at the endoplasmic reticulum caspase-12 for type III apoptosis. Once the caspase cascade is initiated, not only autopro- teolytic activation of caspases occurs after facilitated oligomerization to enhance the pro-apoptotic signal. There is also a caspase cross talk leading to activation of other caspases. The majority of proteolytic cleavage events that manifest the apoptotic phenotype are mediated by "effector" caspases, such as caspase-3 and caspase-7, which become fully activated after endoproteolysis by upstream "initiator" caspases. Caspase-3 is the most important effector caspase. Its activation hallmarks the point of no return in apoptosis signaling (Earnshaw et al., 1999).

\section{METHODOLOGY FOR ANALYSIS OF CASPASES IN HUMAN SPERM \\ Fluorimetric Assays: Flow Cytometry and Fluorescence Microscopy}

The detection of activated caspases in living spermatozoa can be performed using fluorescence labeled inhibitors of caspases (FLICA ${ }^{\mathrm{TM}}$ ). It allows investigating caspase activation in semen samples with regard to the single cell. The FLICA ${ }^{\mathrm{TM}}$ reagent is comprised of 3 segments-it includes a green (FAM = carboxyfluorescein) fluorescent label; an amino acid peptide inhibitor sequence targeted by the active caspase; and a fluoromethylketone group (FMK), which acts as a leaving group and forms a covalent bond with the active enzyme (Ekert et al., 1999). Table 1 gives an overview on the current available caspase inhibitors based on this principle.

Fluorescence labeled inhibitors of caspases are cell permeable and noncytotoxic. Active caspases will form a covalent bond with the reagent and retain the fluorescent signal within the cell. Any unbound inhibitor leaves the sperm (Vaux and Korsmeyer, 1999). In somatic cells various studies proved the association of fluorescence labeled caspase inhibitors with other parameters of apoptosis signaling, e.g., the disruption of 
TABLE 1. Overview on available Caspase inhibitors

\begin{tabular}{lll}
\hline Caspase & \multicolumn{1}{c}{ Substrate } & \multicolumn{1}{c}{$\begin{array}{c}\text { Peptide inhibitor sequence } \\
\text { Carboxyfluorescein analog of: }\end{array}$} \\
\hline Pan-Caspase & FAM-VAD-FMK & benzyloxycarbonyl-valylalanylaspartic acid fluoromethyl ketone \\
Caspase-1 & FAM-YVAD-FMK & benzyloxycarbonyl-tyrosylvalylalanylaspartic acid fluoromethyl ketone \\
Caspase-2 & FAM-VDVAD-FMK & benzyloxycarbonyl-valylaspartylvalylalanylaspartic acid fluoromethyl ketone \\
Caspase-3\&7 & FAM-DEVD-FMK & benzyloxycarbonyl-aspartylglutamylvalylaspartic acid fluoromethyl ketone \\
Caspase-6 & FAM-VEID-FMK & benzyloxycarbonyl-valylglutamylisoleucylaspartic acid fluoromethyl ketone \\
Caspase-8 & FAM-LETD-FMK & benzyloxycarbonyl-leucylglutamylthreonylaspartic acid fluoromethyl ketone \\
Caspase-9 & FAM-LEHD-FMK & benzyloxycarbonyl-alylglutamylvalylaspartic acid fluoromethyl ketone \\
Caspase-10 & FAM-AEVD-FMK &
\end{tabular}

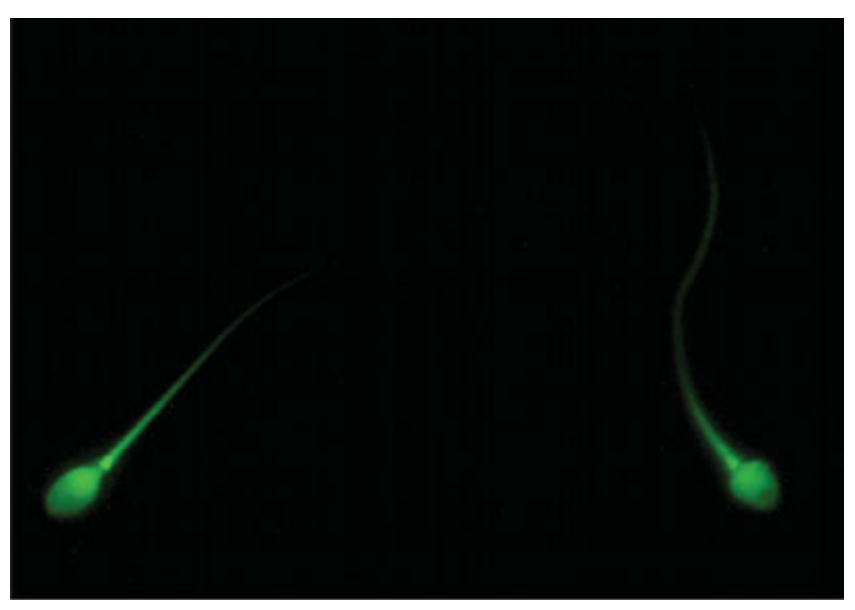

Fig. 2. Image of fluorescence microscopy of activated caspase-3 in human sperm. The active enzyme is detected by a fluorimetric assay (Caspase-3 FLICA $^{\mathrm{TM}}$ ).

the transmembrane mitochondrial potential, the externalization of phosphatidylserine (EPS) and the DNA fragmentation rate (Amstad et al., 2001; Bedner et al., 2000; Pozarowski et al., 2003; Smolewski et al., 2001).

Inhibition of caspase activation by specific inhibitors does not result in diminished FLICA fluorescence (Amstad et al., 2001). This indicates a rather complex binding of caspase inhibitors to the active center of caspases (Pozarowski et al., 2003). However, the same study proves the high specificity of FLICA for detection of caspase activation and recommends the use of fluorescence labeled inhibitors of caspases in context of apoptosis signal transduction (Pozarowski et al., 2003).

The detection of activated caspases by application of FLICA's can be performed either with fluorescence microscopy (Fig. 2) or using flow cytometry in spermatozoa (Brugnon et al., in press-a). Fluorescence labeled inhibitors of caspases are commercially available from various companies (e.g., Immunochemistry Technologies, Bloomington, MN; Clontech Laboratories, Palo Alto, CA; Calbiochem, San Diego, CA). The application is similar and easy to perform. In brief, a 150-fold stock solution of the lyophilisized inhibitor is prepared in dimethyl sulfoxide (DMSO). It is further diluted in phosphate buffered saline (PBS) to make a 30-fold working solution. The prepared sperm aliquots are incubated at $37^{\circ} \mathrm{C}$ for 20 min with $10 \mu \mathrm{L}$ of the working solution, washed with the rinse buffer provided with the kit (alternatively PBS) and analyzed. Human neutrophils $\left(5 \times 10^{6}\right.$ cells) treated with $1 \mathrm{mM}$ cycloheximide for $6 \mathrm{~h}$ can be used as positive controls for induction of apoptosis (Cohen et al., 1992). The negative controls are processed identically, except that the stain was replaced with $10 \mu \mathrm{L}$ PBS.

The commercially available assays are easy to perform in a reasonable time frame. Although immediate analysis of the fluorescence signals is recommended, stability analyses revealed, that it is possible to evaluate activation of caspase- 3 up to 10 days after staining of human sperm (Grunewald et al., 2008a).

All of the investigated caspases could be detected within neat and cryopreserved semen samples. The active enzymes were localized predominantly in the postacrosmal region (CP-8, -1 and -3) (Marchetti et al., 2004; Paasch et al., 2004a) or in the midpiece (Almeida et al., 2005). In contrast the mitochondrial initiator caspase was located only in the midpiece (CP-9). The second finding serves as an internal quality control for the specificity of enzyme detection with fluorimetric assays (Paasch et al., 2004a).

\section{Colorimetric Assays}

Colorimetric assays for detection of activated caspases are based on the same principle as the fluorescence labeled inhibitors of caspases (Table 1). Only the labeling is different, a chromophore is coupled to the caspase inhibitor and becomes fluorescent upon cleavage by the active protease. Most often $p$-nitroaniline is used as chromophore (CaspACE ${ }^{\mathrm{TM}}$ Assay System, Promega, Madison, WI) and produces a yellow color that is monitored by a spectrophotometer at $405 \mathrm{~nm}$. Among others further manufacturers are MBL International (Woburn, MA), R\&D Systems (Minneapolis, MN) or Calbiochem (San Diego, CA). The spectrophotometric evaluation however does not allow the investigation of single cells and although the method probably is suitable, in ejaculated spermatozoa no data on colorimetric caspase detection are currently published.

\section{Western Blot: Adaption of Protocols for Spermatozoa}

Western blotting allows the detailed analysis of caspase pro-forms as well as inactivated and activated caspase forms. Caspase- $1,-3,-7,-8,-9$, and -12 could be demonstrated in human sperm by immunoblot studies (Paasch et al., 2005; Taylor et al., 2004; Wang et al., 2003; Wuendrich et al., 2006). However, due to the small cytoplasmic compartment the detection of caspases in human sperm might be quite difficult. One study denied the presence of caspase- 3 in human sperm due to negative western blot results (De Vries 
et al., 2003). As a consequence, the externalization of phosphatidylserine was misinterpreted as not caspasedependant and not associated with apoptosis in sperm. The standard western blot protocols need to be adapted for investigation of human sperm. Particularly application of appropriate amounts of sperm for protein extraction and sufficient sperm lysis are necessary. The addition of DNAse (2\% benzonase, Merck, Bad Soden, Germany) after sperm lysis is useful to reduce the high viscosity caused by high contents of interfering nucleic acids. The protocol developed by Wuendrich et al. (2006) is recommended for the detection of caspase- $1,-3,-8,-9$, and 12 .

\section{Immunohistochemistry: Caspase Detection in Testicular Tissue}

Immunohistochemistry was used in several studies to investigate caspases in testicular tissue (Giampietri et al., 2005; Hikim et al., 2003; Kim et al., 2007; Pentikainen et al., 1999; Tesarik et al., 2002; Zheng et al., 2006).

For immunohistochemical investigations of testicular tissues two options exist: either cryocut tissue sections (working with almost all antibodies) or paraffin-embedded tissue sections (working with paraffin-reactive antibodies only). As a speciality of testicular samples, Bouin's solution should be applied for optimal fixation when preparing the paraffin-embedded tissue sections.

Interestingly testicular sperm cannot be stained by immunohistochemistry, at least, when standard protocols are applied. This might be due to the minimal amount of cytoplasm at this stage of germ cell maturation, when spermatids eliminate their bulk cytoplasm and undergo terminal differentiation.

During germ cell proliferation and maturation from diploid spermatogonia to mature haploid spermatozoa a number of the developing germ cells are depleted by apoptosis before reaching maturity, even under physiological conditions (Billig et al., 1995). Under pathological circumstances, caspase activation and germ cell apoptosis can be induced by a wide variety of cofactors, e.g., heat, irradiation, ischemia, toxicants as well as withdrawal or exposure to supraphysiological levels of different hormones (Said et al., 2004).

\section{Gene Expression Level: Detection of Caspase-3 mRNA in Sperm}

The presence of RNA's in spermatozoa is a result of transcription during spermatogenesis and can be seen as a historic fingerprint of the individual spermatogenesis. A recent study (Ostermeier et al., 2004) could demonstrate that spermatozoal RNAs complement at least in part the oocyte RNAs after fertilization. Only one study investigated the presence of caspase-3 mRNA in human sperm and although the presence of caspase-3 mRNA was shown in sperm from fertile, healthy donors, one can only speculate on the functional aspect (Grunewald et al., 2005b). The missing translational ability of sperm suggests a translation of this mRNA during or after the fertilization process within the oocyte.

\section{TRIGGERS AND INHIBITORS OF CASPASE ACTIVATION IN HUIMAN SPERM Specific Induction of Various Apoptosis Pathways}

In human ejaculated spermatozoa the presence of Fas-mediated cell death (type-I apoptosis) is still controversially discussed. While recent studies deny the presence of the Fas receptors on human sperm (Perticarari et al., 2008), earlier studies detected it on the surface of subsets of ejaculated spermatozoa using various techniques like western blotting and immuncytochemistry (Grunewald et al., 2001; Sakkas et al., 1999). However, their functional impact is unclear. In one study, Fas was detected in fewer than $10 \%$ of spermatozoa obtained from healthy donors and in more than $10 \%$ of spermatozoa from donors with abnormal spermiogram parameters. The fact that some ejaculated spermatozoa are Fas positive indicates that in some men with abnormal semen parameters, an "abortive apoptosis" has taken place (Sakkas et al., 1999). The initiation of type-I apoptosis using an inducing Fas antibody did not significantly increase levels of caspase- 3 and -8 in ejaculated sperm. Furthermore, it had no effect on caspase- 9 and -1 activation (Grunewald et al., 2005c). These results suggest that receptor mediated (type-I) caspase activation may not have a functional relevance in human ejaculated spermatozoa.

In contrast, due to the compartmentalization in the midpiece of spermatozoa their mitochondria are especially susceptible to various stimuli such as cellular stress caused by cryopreservation, oxidative stress and increased intracellular calcium levels. Certain studies proved that the classical mitochondria-derived apoptotic signaling-cascade is activated in spermatozoa. This evidence included the presence of caspase- 9 and caspase- 3 activation as well as the fact that the mitochondrial membrane depolarizes in response to the aforementioned stimuli (Oehninger et al., 2003; Paasch et al., 2003; Wang et al., 2003). The type-II apoptosis inductor betulinic acid significantly triggered caspase9 and caspase- 3 activation. Caspase- 8 was activated to a certain level, most probably as a result of caspase "cross talk" (Grunewald et al., 2005c; Paasch et al., $2004 b)$. These results were confirmed in a very recent study (Perticarari et al., 2008) suggesting that spermatozoal mitochondria are extremely susceptible to specific agonists of apoptosis. Betulinic acid molecules are currently being used experimentally in anticancer treatments, their side effects on the male reproductive system should be carefully considered.

The studies highlight the extreme susceptibility of spermatozoal mitochondria to specific agonists of apoptosis and downplay the functional relevance of the Fas/Fas receptor in mediating caspase activation in human ejaculated spermatozoa. The role of calciumdependant, endoplasmic reticulum related apoptosis in sperm needs further clarification.

\section{Oxidative Stress}

Although the negative impact of oxidative stress on the sperm DNA integrity is proven by several studies, its impact on sperm caspase activation is not fully clarified. One study measuring the level of oxidative stress and caspase- 9 and -3 activation in sperm from 
males with idiopathic infertility documented a positive correlation of oxidative stress and caspase activation (Wang et al., 2003), this relationship could not be verified in induction studies. Oxidative stress-induced apoptosis appears to be caspase-independent. In detail, incubation with low and high concentrations of $\mathrm{HOCl}$ and $\mathrm{H} 2 \mathrm{O} 2$ respectively did not result in caspase-1, -3 , -8 , and -9 activation in human sperm (Grunewald et al., 2005c; Martinez-Pastor et al., 2009; Taylor et al., 2004). Nevertheless, a recent study showed caspaseactivation in boar spermatozoa after incubation with extremely high levels of NO (Moran et al., 2008).

\section{Cryopreservation-Induced Caspase Activation}

Cryopreservation of human semen is the most commonly accepted method of preserving male reproductive capacity. Cryopreserved spermatozoa may be used in assisted reproductive techniques (ART), especially in cases wherein a patient is diagnosed with cancer and the treatment may render him infertile. The indications for sperm cryobanking have been greatly expanded by recent breakthroughs in ART, in which immotile but viable sperm can be used successfully for oocyte fertilization through intracytoplasmic sperm injection (ICSI). Cryopreservation leads to a significantly increased percentage of sperm showing activation of all types of caspases. Using a pan-caspase (CP-1 to -9) inhibitor, a cryopreservation and thawing related caspase activation was detected in $25.9 \%$ of human sperm from healthy, fertile donors (neat: $21.8 \%$ versus cryopreserved-thawed: $47.7 \%, P<0.01$ ). The caspase activation in cryopreserved-thawed sperm was associated with externalization of phosphatidylserine at the sperm membrane, a sign of membrane damage which is usually seen in apoptotic cells (Grunewald et al., 2001). The highest cryopreservation-induced increase in caspase activation was found in sperm positive for active CP-3 $(+32.6 \%)$ followed by active CP-8+ sperm $(+30.5 \%)$, active CP-9+ sperm $(+22.2 \%)$ and active CP-1+ sperm $(+15.5 \%)$ underlining the central role of the effector caspase-3 (Paasch et al., 2004a). Hence caspase-3 marks a "point of no return" in the apoptosis signaling cascade, the pronounced activation of the protease by cryopreservation and thawing displays the deleterious influence of this process on sperm. Moreover, cryopreservation and thawing related caspase activation is significantly increased in semen samples from subfertile males (Grunewald et al., 2005d). The increase in caspase activation is dependant on the applied sperm preparation and cryopreservation protocol (Grunewald et al., 2005d). Comparative studies showed clearly a strong caspase activation when sperm were shock frozen $(>90 \%$ of sperm contained active caspases), while stepwise cryopreservation protocols induce caspase activation to a significantly lower extent (Said et al., 2004). Density gradient centrifugation (DGC) enables selection of sperm with improved cryo-tolerance. Recent studies underline this finding by measuring significantly reduced levels of caspase-3 in DGC prepared samples. The effect can be further enhanced by application of specific sperm selection methods like Annexin-V magnetic activated cell sorting and Annexin-V glass wool (Grunewald et al., 2007). Therefore, monitoring caspase-3 activation should be used for further optimization of cryopreservation and thawing protocols.

Caspase activation following the cryopreservation and thawing process was also seen in animal germ cells, e.g., in bovine (Martin et al., 2004, 2007) and equine spermatozoa (Brum et al., 2008; Ortega-Ferrusola et al., 2008). However, supplementation of cryopreservation media with caspase inhibitors does not improve the cryosurvival rates of sperm (Peter et al., 2005). The study was performed on canine sperm, but it is likely that the results can be transferred to human sperm.

\section{Capacitation-Related Inactivation of Caspases}

The impact of capacitation on apoptosis-related signal transduction in human sperm was only subject to very few investigations. While some studies observed the externalization of phosphatidylserine (in somatic cells marker of terminal apoptosis) under capacitating conditions (De Vries et al., 2003; Gadella and Harrison, 2002 ), it could not be verified in recent studies (Grunewald et al., 2006a; Muratori et al., 2004). Capacitation of the mature sperm fraction obtained by density gradient centrifugation led to a significant reduction of sperm with active apoptosis signaling. Remarkably, the inactivation was more pronounced at the level of initiator caspases (CP-9) than effector caspases (CP-3). This underlines, that in sperm the activation of the effector caspase-3 marks a "point of no return" in the apoptosis signaling cascade as known from somatic cells (Green and Amarante-Mendes, 1998), while activation of initiator caspases is a reversible process. In concordance with the high susceptibility of sperm to stimulators of mitochondria-associated apoptosis (Grunewald et al., 2005c; Paasch et al., 2004b) the capacitation-induced inhibition of apoptosis signaling was most prominent in the mitochondria; their transmembrane potential integrity was preserved during the capacitation process. Intact mitochondria are essential for energy supply and the basis of sperm motility (Marchetti et al., 2004). Possibly, the improved mitochondrial function allows the hyperactivated motility during capacitation.

The negative association between capacitation (measured by tyrosine phosphorylation, hyperactivates motility and chlortetracycline assay) and caspase activation (CP-3 and -9) was consistently observed for all investigated parameter emphasizing the inverse character of both processes (Grunewald et al., 2005a).

\section{Association of the Sperm Maturity Status and Caspase Activation}

Incomplete maturation of human ejaculated spermatozoa is associated with an increase of initiator and effector caspase activity (Paasch et al., 2003). While the mature sperm subpopulation taken from fertile donor ejaculates contained only $29.4 \%$ (caspase-8), $31.3 \%$ (caspase-9) and $30.3 \%$ (caspase-3) positive sperm the immature subset contained $53.6 \%, 53.1 \%$, and $53.6 \%$ spermatozoa caspase- $8,-3$, and 9 positive sperm respectively $(P<0.01)$. This caspase activation is also associated with the disruption of mitochondrial membrane potential in the immature sperm subpopulation. However, the activated apoptotic process does not immediately affect the levels of DNA fragmentation 
(Paasch et al., 2004c). Particularly cytoplasmic droplets of immature sperm contain activated caspases (Paasch et al., 2003) supporting the theory of abortive apoptosis following incomplete spermatogenesis (Sakkas et al., 1999). In addition, the presence of the antiapoptotic regulator protein bcl-xL in mature sperm reduces caspase-3 activation (Cayli et al., 2004). Recent studies proved the decreased activity of caspase-3 in mature sperm by double probing using aniline blue and caspase-3 immunostaining on the same slide (Sati et al., 2008). Reaching maturity may implicate a deactivation of the apoptosis-signaling cascade in human sperm.

\section{FUNCTION AND RELEVANCE OF CASPASE ACTIVATION IN SPERMATOZOA \\ Impact of Caspase Activation on Conventional Sperm Parameters}

Although in nonapoptotic circumstances in somatic cells caspase- 8 is known as promoter of cell motility (Helfer et al., 2006), in sperm several studies proved a negative correlation of caspase- $3,-8$, and -9 activation and progressive motility. This was true for fresh and cryopreserved-thawed semen samples from donors as well as infertility patients (Grunewald et al., 2001; Marchetti et al., 2004; Paasch et al., 2003, 2004a; Taylor et al., 2004; Said et al., 2006; Weng, 2002). In addition, using a fluorescence labeled inhibitor of caspase $1-9$, a negative correlation of caspase activation and sperm concentration was found in semen samples of males attending an IVF program (Marchetti et al., 2004). Only a single investigation failed to detect the negative impact of caspase activation on sperm motility (Perticarari et al., 2007). However, although the latter study did include 68 semen samples, the patients group was not well defined.

Several studies investigated the relationship between sperm morphology and apoptosis (Aziz et al., 2007; Chen et al., 2006; Gandini et al., 2000; Ricci et al., 2002; Sakkas et al., 2002; Shen et al., 2002; Siddighi et al., 2004). The design of these studies might not have facilitated the accurate distinction between moribund or necrotic sperm and motile sperm expressing apoptotic markers. Other studies correlated sperm morphology in neat semen with apoptosis in selected motile sperm subpopulation obtained after swim-up or double gradient centrifugation techniques (Almeida et al., 2005; Benchaib et al., 2003; Muratori et al., 2003; Weng et al., 2002). All these studies applied different criteria for the assessment of sperm morphology including WHO 1992 standards (Muratori et al., 2003), WHO 1999 standards (Almeida et al., 2005; Benchaib et al., 2003; Weng et al., 2002) and the Tygerberg's strict criteria (Chen et al., 2006; Sakkas et al., 2002; Siddighi et al., 2004; Weng et al., 2002).

For instance normal morphology in semen was found to correlate negatively with caspase-3 activation measured in swim-up preparation but not in whole semen (Almeida et al., 2005). A significant negative relationship between the proportions of apoptotic sperm and the proportions of sperm with normal morphology in semen applying the Tygerberg's strict criteria (Kruger et al., 1988) has been reported (Chen et al., 2006). In another study normal sperm morphology assessed in the swim-up preparations correlated negatively with chromatin fragmentation only in teratozoospermic semen samples but not in normospermic ones as determined by the WHO 1992 criteria (Muratori et al., 2003). Finally, normal morphology applying the strict criteria in sperm preparations was reported to correlate inversely with caspase-3 activation, but had not always a relationship with mitochondrial transmembrane integrity. Detailed analyses applying the sperm deformity index (SDI) score as a novel expression of the quality of sperm morphology became recently available (Aziz et al., 2007). The SDI has been shown to be a more powerful predictor of male fertility and of in vitro fertilization outcome compared to the assessment of the proportion of sperm with normal morphology (Aziz et al., 1996). The authors found a significant relationship between midpiece defects and caspase-3 activation. This is in concordance with the localization of active caspase- 3 in the post acrosomal part in mature sperm (Paasch et al., 2004a) and in the midpiece where mitochondria and residual cytoplasm reside (Weng et al., 2002).

\section{Relation of Caspase Activation and Subcellular Sperm Apoptosis Parameter}

Caspase activation is a central part of the apoptosis signaling cascade in somatic cells (Cohen, 1997) and the investigation of this association in sperm was subject of several studies.

In human sperm the activation of various initiator and effector caspases correlated well with each other $(r>0.5, P<0.01)$ indicating not only a functional cascade of the proteases, but also auto-activation of all subtypes of caspases once the signaling cascade is stimulated (Paasch et al., 2004a). While most studies focused on "typical" apoptosis-related caspases, one study observed a less pronounced correlation of caspase- 9 and -3 to caspase- 1 activation in cryopreserved semen samples (Paasch et al., 2004a) underlining other possible functions of the protease in human sperm than apoptosis signaling.

The exceptional susceptibility of ejaculated spermatozoa to mitochondria-associated apoptosis due to the compartmentalization of the mitochondria in the midpiece region (Paasch et al., 2004b) is displayed by a close relationship of disruption of the transmembrane mitochondrial potential (TMP) and caspase activation. Several studies proved the strong positive correlation in human sperm from fertile donors and infertility patients (Grunewald et al., 2005c, in press; Marchetti et al., 2004; Said et al., 2006) Also, the cryopreservation and thawing related activation of caspases is accompanied by disruption of the transmembrane mitochondrial potential (Marchetti et al., 2004; Paasch et al., 2004c). The latter was also documented in equine spermatozoa (Ortega-Ferrusola et al., 2008). Specific inducers of mitochondria-associated apoptosis like betulinic acid trigger activation of the mitochondriarelated initiator-caspase (CP-9) and TMP disruption as well as activation of the downstream effector caspase-3 (Paasch et al., 2004b). Diminished integrity of the transmembrane mitochondrial potential marks the altered mitochondrial function. Possibly, the observed effects in sperm like loss of motility and consequently decreased oocyte penetration capacity are not only a result of the reduced mitochondrial energy production, 
but also due to the accompanying caspase activation (Grunewald et al., 2008b).

The relationship of externalization of phosphatidylserine (EPS) and caspase activation in sperm cells is a controversially discussed issue as it refers to the relationship of externalization of phosphatidylserine and sperm apoptosis itself. In somatic cells externalized phosphatidylserine at the outer plasmamembrane is a sign of the terminal phase of apoptosis and labels the cell for phagocytosis (Fadok et al., 1998). In human spermatozoa however, the head is covered by the acrosome. Although already early studies showed a close association of EPS and caspase 1-9 activation in neat and cryopreserved-thawed sperm (Grunewald et al., 2001; Paasch et al., 2003) other studies linked the externalization of phosphatidylserine to the capacitation process (De Vries et al., 2003). However, the latter study failed to detect caspase- 3 by western blotting at all most probably due to technical problems which in turn does not allow any speculation on the association of caspase activation and externalization of phosphatidylserine from their side. Moreover, further investigations could not detect EPS as marker of capacitation (Muratori et al., 2004). On the other hand induction of acrosome reaction by calcium ionophore A23187 was shown to result in increased numbers of sperm with externalized phosphatidylserine (Martin et al., 2005). Until now it could not been clarified completely if this is due to the presence of phosphatidylserine at the inner leaflet of the acrosome or if the nonphysiological induction of the acrosome reaction (calcium ionophore A23187 is also used as apoptosis inductor in somatic cells) might have deleterious and apoptosis-stimulating side effects. Separation of EPS-negative and -positive sperm using Annexin-V magnetic activated cell sorting did not affect the number of spontaneously acrosomereacted sperm (control and both fractions $<5 \%$ ). In contrast, EPS-negative sperm showed superior capacity to undergo capacitation and (after induction) acrosome reaction (Grunewald et al., 2006a). Furthermore, studies using the same separation system showed a positive correlation of EPS and caspase-1, -3, -8 , and -9 activation and a depletion of caspase-positive sperm in the EPS negative fraction (Grunewald et al., 2001, 2003, 2004a; Said et al., 2005). Those results were confirmed by investigations on the sperm fertilizing potential, which was found to be reduced in EPS+ sperm with activated caspases (Grunewald et al., 2008b; Said et al., 2006) and recently published correlation analyses (Kotwicka et al., 2008).

\section{Increased Sperm DNA Fragmentation Due to Caspase Activation?}

Sperm DNA fragmentation negatively affects spontaneous conception as well as the outcome of IVF and ICSI (Zini et al., 2008). Several models exist to explain the origin of DNA fragmentations in human sperm. Sperm DNA fragmentations can result from insufficient nuclear remodeling during spermatogenesis. Alternatively DNA fragmentations might be remnants of abortive apoptosis processes in a deficient spermatogenesis. In addition post-testicular affections of sperm, e.g., due to oxidative stress can induce DNA fragmentations (Muratori et al., 2006). Apoptosis-related DNA fragmentation as seen in somatic cells is in part a result of caspase-3 activation. The main effector protease of the apoptosis signaling cascade cleaves and thereby inactivates poly-(ADP-ribose)-polymerase (PARP), which in turn negatively affects DNA repair mechanisms (Cohen, 1997). A recently published study detected a positive correlation of caspase-3 activation and PARP cleavage in human sperm (Mahfouz et al., in press). Although a direct link between caspase activation and DNA fragmentations has not been proven in human sperm yet, it might be the explanation of the repeatedly documented positive correlation of the percentage of spermatozoa with fragmented DNA and caspase-3 activation. The association was weak in fertile donors (Said et al., 2006), but more pronounced in semen samples from infertile patients $(r=0.6, P<$ 0.01) (Marchetti et al., 2004).

\section{Influence of Caspase Activation on Sperm Penetration Capacity and Sperm Chromatin Decondensation}

Only very few studies investigated the impact of caspase activation on sperm fertilizing potential.

In male partners of couples included in an IVF program, a negative correlation of pan-caspase activation and fertilization rates after IVF $(r=0.38, P<0.05)$ was demonstrated (Marchetti et al., 2004).

Own investigations used animal models like the zona free hamster oocyte penetration assay (SPA, simulation of IVF) and intracytoplsmic sperm infection into hamster oocytes (H-ICSI, simulation of ICSI) to determine the sperm penetration capacity and sperm chromatin decondensation. In semen samples derived from healthy donors, activation of the executioner caspase-3 was significantly negatively associated with the percentage of penetrated oocytes $(r=-0.61, P<0.01)$ and to a lesser extent with the number of sperms per penetrated oocyte $(r=-0.44, P<0.01)$. This is underlined by similar effects measured at another crucial point of apoptosis signaling in human ejaculated sperm. Disruption of the mitochondrial transmembrane potential showed a significant negative correlation with the percentage of penetrated hamster oocytes and the amount of penetrated sperm per penetrated hamster oocyte $(r=0.67$ and $r=0.48$, respectively, $P<0.01$ ). The results of the significant interrelation of caspase- 3 activation and oocyte penetration were confirmed in a population of 76 unselected male partners of infertile couples. While semen samples with normal sperm penetration assay values contained $23.0 \%$ caspase-3 positive germ cells, semen samples showing impaired oocyte penetration (SPA $<20 \%)$ contained $41.4 \%$ sperm with activated caspase-3 $(P<0.01)$ (Grunewald et al., 2008b).

However, using healthy donor sperm only a weak negative correlation of caspase activation and sperm chromatin decondensation rates after H-ICSI could be detected ( $r=-0.31, P=0.01$ ) (Said et al., 2006), but recently published data on infertility patients with oligoasthenoteratozoospermia syndrome and high caspase-3 activation levels revealed a stronger relationship ( $r=0.45, P<0.01)$ of both parameters in ICSI simulation (Grunewald et al., in press). 


\section{Caspase Activation in Sperm From Infertility Patients}

Levels of caspase activity are higher in semen samples from subfertile patients compared to healthy donors. In a first study, caspase enzymatic activity was higher in patients than in donors in both low and high motility fractions. A significant positive correlation between in situ active caspase-3 in the sperm midpiece and DNA fragmentation was observed in the low motility fractions of patients, suggesting that caspase-dependent apoptotic mechanisms could originate in the cytoplasmic droplet or within mitochondria and function in the nucleus (Weng et al., 2002).

Quantitative Western blot analyses confirmed the higher activation levels of caspase-1, -8 and -9 in infertility patients compared to fertile donors on protein level. This effect raised with increasing glycerol concentration from $7 \%$ to $14 \%$ (Wuendrich et al., 2005). The higher level in activation of caspases in cryostored spermatozoa of infertility patients may indicate that these cells have a lower cryotolerance and a higher susceptibility to caspase activation than the spermatozoa of donors (Grunewald et al., 2005d). Analysis of caspase-3 activation in semen samples from subfertile males with oligoasthenoteratozoospermia and teratozoospermia revealed elevated levels of the active protease when compared to samples from healthy, fertile donors (Grunewald et al., in press). This corresponds to an earlier study showing a positive relationship of caspase-3 activation and pathological sperm morphology (Aziz et al., 2007). Very recently, increased levels of caspase-3 activation were found in thawed epididymal $(57.5 \%)$ and testicular (96.0\%) spermatozoa of patients with congenital bilateral absence of the vas deferens correlating negatively with ICSI fertilization, good morphology embryo at day 2 , clinical pregnancy, and implantation rates (Brugnon et al., in press-b). Moreover, the reduced sperm quality in patients with urogenital infections might be mediated by induction of apoptosis. Coincubation with Chlamydia trachomatis lipopolysaccharide led to an activation of sperm caspases (Eley et al., 2005).

\section{THERAPEUTIC APPROACHES TO DEPLETE SPERM WITH ACTIVATED CASPASES}

Standard sperm preparation techniques are based on outcome measures as sperm yield, sperm motility, viability, and morphology. Although the conventional analysis gives considerable information, it does not assess the presence of deregulated programmed cell death (apoptosis) in spermatozoa, which may be partially responsible for the low fertilization and implantation rates seen with ART.

\section{Potential of Standard Sperm Preparation Techniques}

Following simple wash of semen samples from fertile donors approximately one third of sperm contained active caspase-3.

In a comparative study the ability of two types of conventional glass wool filtration systems to deplete sperm with activated caspase-3 has been investigated recently. Raw glass wool had a clear negative impact on semen samples. Not only caspase-3 activation but also the disruption of mitochondrial membrane potential exceeded the levels measured in controls. Only when applied to cryopreserved samples was there a minor filtration effect in terms of apoptosis markers (Henkel and Schill, 2003). In concordance with studies on conventional sperm parameters raw glass wool may not be used for sperm preparation prior to ART (Grunewald et al., 2007). The application of the commercially available SpermFertil ${ }^{\mathbb{R}}$ glass wool was perfectly tolerated by sperm, but the reduction of sperm with activated effector caspase-3 was only slight and failed to reach statistical significance. However, in semen samples with higher content of caspase positive sperm, e.g., following cryopreservation and thawing a significant depletion effect was observed (Paasch et al., 2007).

In contrast several studies validated the potential of double density gradient centrifugation to reduce the amount of caspase-positive sperm significantly (Said et al., 2005, 2006). The investigations were performed in sperm derived from healthy male donors (Grunewald et al., in press), infertility patients (Brum et al., 2008; Martí et al., 2006) and animals (ram and equine spermatozoa) (Blanc-Layrac et al., 2000). In detail, double density gradient centrifugation is able to reduce the number of sperm showing caspase-3 activation to $15-20 \%$ in neat donor semen samples and to $43.5 \%$ in semen specimens from infertility patients. Concordantly other studies proved the ability of the method to decrease the amount of DNA-fragmented sperm by density gradient centrifugation (Gandini et al., 1999). The double density gradient centrifugation related depletion effect of caspase-positive sperm might be explained by the elimination of the majority of immature spermatozoa (Grunewald et al., in press; Said et al., 2006), which contain high levels of active caspases (Paasch et al., 2004b). However, compared to specific preparation methods based on selection of nonapoptotic sperm density gradient centrifugation is less powerful to deplete caspase-positive sperm (Dominguez et al., 1999).

The swim-up procedure uses the active motion of spermatozoa. Intact moving cells are swimming out of a pellet derived by a simple washing step into an overlaid media for 30-60 min. Highly motile, morphologically intact spermatozoa are enriched in the absence of other cells, proteins and debris within the supernatant. Hence such sperm contain lower amounts of active caspases, their enrichment resulted as expected in decreased levels of caspase activation (Almeida et al., 2005). Concordantly, swim up fractions from infertility patients contained relatively low numbers of caspase-3 positive sperm (Kotwicka et al., 2008). However, the same study indicated that swim up preparations from semen samples with poor morphology contain higher amounts of sperm with active caspase-3. In healthy donors swim up resulted in a significant depletion $(-15 \%)$ of caspase-3 positive sperm.

In subfertile males, semen preparation with density gradient centrifugation followed by swim up was also reported to result in a significant depletion of spermatozoa with activated apoptosis-related signaling. The high amounts of spermatozoa with active caspase-3 and disrupted transmembrane potential in semen samples from infertility patients were significantly 
TABLE 2. Results of separation studies using Annexin V-based techniques alone or in combination with density gradient centrifugation

\begin{tabular}{|c|c|c|c|c|}
\hline \multirow[b]{2}{*}{ Separation method/samples } & \multicolumn{4}{|c|}{ Percentage of caspase-3 positive sperm } \\
\hline & $\begin{array}{c}\text { Non-separated } \\
\text { semen }\end{array}$ & $\begin{array}{l}\text { EPS-negative } \\
\text { fraction }\end{array}$ & $\begin{array}{l}\text { EPS-positive } \\
\text { fraction }\end{array}$ & Study \\
\hline MACS/neat sperm of fertile donors & $18.1 \pm 7.5$ & $13.2 \pm 6.7$ & $50.8 \pm 9.9$ & Paasch et al., 2004a \\
\hline DGC + MACS/neat sperm of fertile donors & $15.2 \pm 10.5$ & $8.8 \pm 5.9$ & $70.5 \pm 18.8$ & Said et al., 2006 \\
\hline MACS/cryopreserved-thawed sperm of fertile donors & $50.7 \pm 5.9$ & $14.3 \pm 6.2$ & $86.7 \pm 4.3$ & Paasch et al., 2004a \\
\hline DGC + MACS/neat sperm of infertility patients & $43.5 \pm 13.8$ & $26.8 \pm 12.3$ & $58.4 \pm 13.8$ & Grunewald et al., in press \\
\hline Annexin V glass wool/neat sperm of fertile donors & $34.4 \pm 3.2$ & $19.5 \pm 2.0$ & $\mathrm{n} / \mathrm{a}$ & Grunewald et al., 2007 \\
\hline $\begin{array}{l}\text { Annexin V glass wool/cryopreserved-thawed sperm } \\
\text { of fertile donors }\end{array}$ & $57.6 \pm 5.3$ & $34.0 \pm 4.1$ & $\mathrm{n} / \mathrm{a}$ & Grunewald et al., 2007 \\
\hline
\end{tabular}

reduced. However, there was a marked interindividual variance of the separation effect. While minimum reduction of sperm with activated caspase-3 was calculated as $<1 \%$ of sperm, a maximum reduction was achieved at $\sim 50 \%$ (Grunewald et al., 2008b).

\section{Annexin V-Based Sperm Separation Techniques}

As mentioned above, the externalization of phosphatidylserine (EPS) is one of the earliest signs of the terminal phase of apoptosis. The specific binding of Annexin V to phosphatidylserine was applied to separate EPS-negative and -positive sperm using the Annexin V MACS and Annexin V coated glass wool (Grunewald et al., 2001, 2007).

All separation studies proved the positive association of the externalization of phosphatidylserine and caspase activation in human sperm. The separated EPSnegative sperm fraction contained significantly reduced amounts of caspases (Grunewald et al., 2007; Paasch et al., 2004a; Said et al., 2006). An overview is given in Table 2.

Various studies clearly indicated that integrating Annexin V MACS as a part of sperm preparation techniques will improve semen quality and cryosurvival rates by eliminating sperm with activated caspases (Grunewald et al., 2006b; Said et al., 2005, 2008). Nonapoptotic spermatozoa prepared by Annexin V MACS display improved routine sperm parameters and decreased apoptosis markers. The higher sperm quality is represented by an increased oocyte penetration potential and cryosurvival rates (Grunewald et al., in press; Said et al., 2006). Thus, the depletion of sperm with active caspases by MACS should be considered to enhance ART success rates.

\section{CONCLUSION}

In ejaculated sperm, activation of caspases is a sign of immaturity and seen particularly in semen samples infertility patients. In mature sperm caspase activation can be observed after specific apoptosis induction, but also under subphysiological conditions like cryopreservation and thawing. During the capacitation process, the caspase cascade is significantly inactivated. Apoptosis-related caspase activation appears to have a negative association with sperm-oocyte penetration and sperm chromatin decondensation after fertilization. The exclusion of sperm presenting with those apoptosis-related features during assisted reproduction may improve success rates of procedures such as intrauterine insemination, in vitro fertilization and intracytoplasmic sperm injection.

\section{REFERENCES}

Almeida C, Cardoso MF, Sousa M, Viana P, Goncalves A, Silva J, Barros A. 2005. Quantitative study of caspase-3 activity in semen and after swim-up preparation in relation to sperm quality. Hum Reprod 20:1307-1313.

Amstad PA, Yu G, Johnson GL, Lee BW, Dhawan S, Phelps DJ (2001) Detection of caspase activation in situ by fluorochrome-labeled caspase inhibitors. Biotechniques, 31:608-610, 612, 614, passim.

Aziz N, Buchan I, Taylor C, Kingsland CR, Lewis-Jones I. 1996. The sperm deformity index: a reliable predictor of the outcome of oocyte fertilization in vitro. Fertil Steril 66:1000-1008.

Aziz N, Said T, Paasch U, Agarwal A. 2007. The relationship between human sperm apoptosis, morphology and the sperm deformity index. Hum Reprod 22:1413-1419.

Bedner E, Smolewski P, Amstad P, Darzynkiewicz Z. 2000. Activation of caspases measured in situ by binding of fluorochrome-labeled inhibitors of caspases (FLICA): Correlation with DNA fragmentation. Exp Cell Res 259:308-313.

Benchaib M, Braun V, Lornage J, Hadj S, Salle B, Lejeune H, Guerin JF. 2003. Sperm DNA fragmentation decreases the pregnancy rate in an assisted reproductive technique. Hum Reprod 18:1023-1028.

Billig H, Furuta I, Rivier C, Tapanainen J, Parvinen M, Hsueh AJ. 1995. Apoptosis in testis germ cells: Developmental changes in gonadotropin dependence and localization to selective tubule stages. Endocrinology 136:5-12.

Blanc-Layrac G, Bringuier AF, Guillot R, Feldmann G. 2000. Morphological and biochemical analysis of cell death in human ejaculated spermatozoa. Cell Mol Biol (Noisy-le-grand) 46:187-197.

Brugnon F, Ouchchane L, Verheyen G, Communal Y, Van der EJ, Tournaye H, Janny L, Grizard G. Fluorescence microscopy and flow cytometry in measuring activated caspases in human spermatozoa. Int J Androl, DOI:10.1111/j.1365-2605.2007.00847.

Brugnon F, Janny L, Artonne C, Sion B, Pouly JL, Grizard G. Activated caspases in thawed epididymal and testicular spermatozoa of patients with congenital bilateral absence of the vas deferens and intracytoplasmic sperm injection outcome. Fertil Steril, DOI:10.1016/j.fertnsert.2008.07.1712.

Brum AM, Sabeur K, Ball BA. 2008. Apoptotic-like changes in equine spermatozoa separated by density-gradient centrifugation or after cryopreservation. Theriogenology 69:1041-1055.

Cayli S, Sakkas D, Vigue L, Demir R, Huszar G. 2004. Cellular maturity and apoptosis in human sperm: Creatine kinase, caspase-3 and Bcl-XL levels in mature and diminished maturity sperm 1. Mol Hum Reprod 10:365-372.

Chen Z, Hauser R, Trbovich AM, Shifren JL, Dorer DJ, Godfrey-Bailey L, Singh NP. 2006. The relationship between human semen characteristics and sperm apoptosis: A pilot study. J Androl 27:112-120.

Cohen GM. 1997. Caspases: The executioners of apoptosis. Biochem J, 326 (Part 1):1-16.

Cohen JJ, Duke RC, Fadok VA, Sellins KS. 1992. Apoptosis and programmed cell death in immunity. Annu Rev Immunol 10:267-293.

Cryns V, Yuan J. 1998. Proteases to die for. Genes Dev 12:1551-1570.

De Vries KJ, Wiedmer T, Sims PJ, Gadella BM. 2003. Caspase-independent exposure of aminophospholipids and tyrosine phosphorylation in bicarbonate responsive human sperm cells. Biol Reprod 68:2122-2134.

Dominguez LA, Burgos MH, Fornes MW. 1999. Morphometrical comparison of human spermatozoa obtained from semen and swim-up methodology. Andrologia 31:23-26.

Earnshaw WC, Martins LM, Kaufmann SH. 1999. Mammalian caspases: Structure, activation, substrates, and functions during apoptosis. Annu Rev Biochem 68:383-424.

Ekert PG, Silke J, Vaux DL. 1999. Caspase inhibitors. Cell Death Differ 6:1081-1086. 
Eley A, Hosseinzadeh S, Hakimi H, Geary I, Pacey AA. 2005. Apoptosis of ejaculated human sperm is induced by co-incubation with Chlamydia trachomatis lipopolysaccharide. Hum Reprod 20:2601-2607.

Fadok VA, Bratton DL, Frasch SC, Warner ML, Henson PM. 1998. The role of phosphatidylserine in recognition of apoptotic cells by phagocytes. Cell Death Differ 5:551-562.

Fuentes-Prior P, Salvesen GS. 2004. The protein structures that shape caspase activity, specificity, activation and inhibition. Biochem J 384:201-232.

Gadella BM, Harrison RA. 2002. Capacitation induces cyclic adenosine $3^{\prime}, 5^{\prime}$-monophosphate-dependent, but apoptosis-unrelated, exposure of aminophospholipids at the apical head plasma membrane of boar sperm cells. Biol Reprod 67:340-350.

Gandini L, Lenzi A, Lombardo F, Pacifici R, Dondero F. 1999. Immature germ cell separation using a modified discontinuous Percoll gradient technique in human semen. Hum Reprod 14:1022-1027.

Gandini L, Lombardo F, Paoli D, Caponecchia L, Familiari G, Verlengia C, Dondero F, Lenzi A. 2000. Study of apoptotic DNA fragmentation in human spermatozoa. Hum Reprod 15:830-839.

Giampietri C, Petrungaro S, Coluccia P, D'Alessio A, Starace D, Riccioli A, Padula F, Palombi F, Ziparo E, Filippini A, De Cesaris P. 2005. Germ cell apoptosis control during spermatogenesis. Contraception 72:298-302.

Green DR, Amarante-Mendes GP. 1998. The point of no return: Mitochondria, caspases, and the commitment to cell death. Results Probl Cell Differ 24:45-61.

Grunewald S, Paasch U, Glander HJ. 2001. Enrichment of non-apoptotic human spermatozoa after cryopreservation by immunomagnetic cell sorting. Cell Tissue Bank 2:127-133.

Grunewald S, Paasch U, Baumann T, Kriegel C, Glander HJ. 2005a. Capacitation related inactivation of caspase signaling cascade in human spermatozoa. In: Kim S-C, Grootegoed JA, Chemes H, editors. Eighth International Congress of Andrology, Medimond, Seoul, South Korea, June 12-16, 2005, pp. 63-66.

Grunewald S, Paasch U, Glander HJ, Anderegg U. 2005b. Mature human spermatozoa do not transcribe novel RNA. Andrologia 37:69-71.

Grunewald S, Paasch U, Said TM, Sharma RK, Glander HJ, Agarwal A. 2005c. Caspase activation in human spermatozoa in response to physiological and pathological stimuli. Fertil Steril, 83 (Suppl 4):1106-1112.

Grunewald S, Paasch U, Wuendrich K, Glander HJ. 2005d. Sperm caspases become more activated in infertility patients than in healthy donors during cryopreservation. Arch Androl 51:449-460.

Grunewald S, Baumann T, Paasch U, Glander HJ. 2006a. Capacitation and acrosome reaction in nonapoptotic human spermatozoa. Ann N Y Acad Sci 1090:138-146.

Grunewald S, Paasch U, Said TM, Rasch M, Agarwal A, Glander HJ. 2006 b. Magnetic-activated cell sorting before cryopreservation preserves mitochondrial integrity in human spermatozoa. Cell Tissue Bank 7:99-104.

Grunewald S, Miska W, Miska G, Rasch M, Reinhardt M, Glander HJ, Paasch U. 2007. Molecular glass wool filtration as a new tool for sperm preparation. Hum Reprod 22:1405-1412.

Grunewald S, Rasch M, Reinhardt M, Baumann T, Paasch U, Glander HJ. 2008a. Stability of fluorochrome based assays to measure subcellular sperm functions. Asian J Androl 10:455-459.

Grunewald S, Said TM, Paasch U, Glander HJ, Agarwal A. 2008b. Relationship between sperm apoptosis signalling and oocyte penetration capacity. Int J Androl 31:325-330.

Grunewald S, Reinhardt M, Blumenauer V, Said TM, Agarwal A, Hmeidan FA, Glander HJ, Paasch U. Increased sperm chromatin decondensation in selected non-apoptotic spermatozoa of patients with male infertility. Fertil Steril, DOI:10.1016/j.fertnstert.2008. 07.1705 .

Helfer B, Boswell BC, Finlay D, Cipres A, Vuori K, Bong KT, Wallach D, Dorfleutner A, Lahti JM, Flynn DC, et al. 2006. Caspase-8 promotes cell motility and calpain activity under nonapoptotic conditions. Cancer Res 66:4273-4278.

Henkel RR, Schill WB. 2003. Sperm preparation for ART. Reprod Biol Endocrinol 1:108.

Hikim AP, Lue Y, Yamamoto CM, Vera Y, Rodriguez S, Yen PH, Soeng K, Wang C, Swerdloff RS. 2003. Key apoptotic pathways for heatinduced programmed germ cell death in the testis. Endocrinol 144:3167-3175.

Kim SK, Yoon YD, Park YS, Seo JT, Kim JH. 2007. Involvement of the Fas-Fas ligand system and active caspase-3 in abnormal apoptosis in human testes with maturation arrest and Sertoli cell-only syndrome. Fertil Steril 87:547-553.

Kotwicka M, Filipiak K, Jedrzejczak P, Warchol JB. 2008. Caspase-3 activation and phosphatidylserine membrane translocation in human spermatozoa: Is there a relationship? Reprod Biomed Online 16:657-663.

Kruger TF, Acosta AA, Simmons KF, Swanson RJ, Matta JF, Oehninger S. 1988. Predictive value of abnormal sperm morphology in in vitro fertilization. Fertil Steril 49:112-117.

Kumar S. 2004. Migrate, differentiate, proliferate, or die: Pleiotropic functions of an apical "apoptotic caspase." Sci STKE 2004:e49.

Li J, Yuan J. 2008. Caspases in apoptosis and beyond. Oncogene 27:6194-6206.

Mahfouz RZ, Sharma RK, Poenicke K, Jha R, Paasch U, Grunewald S, Agarwal A. Evaluation of poly(ADP-ribose) polymerase cleavage (cPARP) in ejaculated human sperm fractions after induction of apoptosis. Fertil Steril, DOI:10.1016/j.fertnstert.2008.02.173.

Marchetti C, Gallego MA, Defossez A, Formstecher P, Marchetti P. 2004. Staining of human sperm with fluorochrome-labeled inhibitor of caspases to detect activated caspases: Correlation with apoptosis and sperm parameters. Hum Reprod 19:1127-1134.

Martí E, Pérez-Pé R, Muino-Blanco T, Cebrián-Pérez JA. 2006. Comparative studies of four different sperm washing methods using apoptosis markers in ram spermatozoa. J Androl 27:746-753.

Martin G, Sabido O, Durand P, Levy R. 2004. Cryopreservation induces an apoptosis-like mechanism in bull sperm. Biol Reprod 71:28-37.

Martin G, Sabido O, Durand P, Levy R. 2005. Phosphatidylserine externalization in human sperm induced by calcium ionophore A23187: Relationship with apoptosis, membrane scrambling and the acrosome reaction. Hum Reprod 20:3459-3468.

Martin G, Cagnon N, Sabido O, Sion B, Grizard G, Durand P, Levy R. 2007. Kinetics of occurrence of some features of apoptosis during the cryopreservation process of bovine spermatozoa. Hum Reprod 22:380-388

Martinez-Pastor F, Aisen E, Fernandez-Santos MD, Esteso M, Maroto-Morales A, Garcia-Alvarez O, Garde J. 2009. ROS generators affect quality parameters and apoptosis markers differently in red deer spermatozoa. Reproduction 137:225-235.

Moran JM, Madejon L, Ortega FC, Pena FJ. 2008. Nitric oxide induces caspase activity in boar spermatozoa. Theriogenology 70:91-96.

Muratori M, Maggi M, Spinelli S, Filimberti E, Forti G, Baldi E. 2003. Spontaneous DNA fragmentation in swim-up selected human spermatozoa during long term incubation. J Androl 24:253-262.

Muratori M, Porazzi I, Luconi M, Marchiani S, Forti G, Baldi E. 2004. AnnexinV binding and merocyanine staining fail to detect human sperm capacitation. J Androl 25:797-810.

Muratori M, Marchiani S, Maggi M, Forti G, Baldi E. 2006. Origin and biological significance of DNA fragmentation in human spermatozoa. Frontiers Biosci 11:1491-1499.

Nicholson DW. 1999. Caspase structure, proteolytic substrates, and function during apoptotic cell death. Cell Death Differ 6:1028-1042.

Oehninger S, Morshedi M, Weng SL, Taylor S, Duran H, Beebe S. 2003. Presence and significance of somatic cell apoptosis markers in human ejaculated spermatozoa. Reprod Biomed Online 7:469-476.

Ortega-Ferrusola C, Sotillo-Galan Y, Varela-Fernandez E, GallardoBolanos JM, Muriel A, Gonzalez-Fernandez L, Tapia JA, Pena FJ. 2008. Detection of "apoptosis-like" changes during the cryopreservation process in equine sperm. J Androl 29:213-221.

Ostermeier GC, Miller D, Huntriss JD, Diamond MP, Krawetz SA 2004. Reproductive biology: Delivering spermatozoan RNA to the oocyte. Nature 429:154.

Paasch U, Agarwal A, Gupta AK, Sharma RK, Grunewald S, Thomas AJ Jr, Glander HJ. 2003. Apoptosis signal transduction and the maturity status of human spermatozoa. Ann N Y Acad Sci 1010:486489.

Paasch U, Grunewald S, Fitzl G, Glander HJ. 2003. Deterioration of plasma membrane is associated with activation of caspases in human spermatozoa. J Androl 24:246-252.

Paasch U, Grunewald S, Agarwal A Glandera HJ. 2004a. Activation pattern of caspases in human spermatozoa. Fertil Steril, 81 (Suppl 1):802-809.

Paasch U, Grunewald S, Dathe S, Glander HJ. 2004b. Mitochondria of human Spermatozoa are preferentially susceptible to apoptosis. Ann N Y Acad Sci 1030:403-409.

Paasch U, Sharma RK, Gupta AK, Grunewald S, Mascha EJ, Thomas AJ Jr, Glander HJ, Agarwal A. 2004c. Cryopreservation and thawing is associated with varying extend of activation of apoptotic machinery in subsets of ejaculated human spermatozoa. Biol Reprod 71:1828-1837.

Paasch U, Grunewald S, Wuendrich K, Jope T, Glander HJ. 2005. Immunomagnetic removal of cryo-damaged human spermatozoa. Asian J Androl 7:61-69.

Paasch U, Grunewald S, Glander HJ. 2007. Sperm selection in assisted reproductive techniques. Soc Reprod Fertil 65 (Suppl):515525. 
Pentikainen V, Erkkila K, Dunkel L. 1999. Fas regulates germ cell apoptosis in the human testis in vitro. Am J Physiol 276:E310-E316.

Perticarari S, Ricci G, Granzotto M, Boscolo R, Pozzobon C, Guarnieri S, Sartore A, Presani G. 2007. A new multiparameter flow cytometric method for human semen analysis. Hum Reprod 22:485-494.

Perticarari S, Ricci G, Boscolo R, De SM, Pagnini G, Martinelli M, Presani G. 2008. Fas receptor is not present on ejaculated human sperm. Hum Reprod 23:1271-1279.

Peter AT, Colenbrander B, Gadella B. 2005. Effect of caspase inhibitors on the post-thaw motility, and integrity of acrosome and plasma membrane of cryopreserved equine spermatozoa. Indian J Exp Biol 43:483-487.

Pozarowski P, Huang X, Halicka DH, Lee B, Johnson G, Darzynkiewicz Z. 2003. Interactions of fluorochrome-labeled caspase inhibitors with apoptotic cells: A caution in data interpretation. Cytometry A 55:50-60.

Ricci G, Perticarari S, Fragonas E, Giolo E, Canova S, Pozzobon C, Guaschino S, Presani G. 2002. Apoptosis in human sperm: Its correlation with semen quality and the presence of leukocytes. Hum Reprod 17:2665-2672.

Said TM, Paasch U, Glander HJ, Agarwal A. 2004. Role of caspases in male infertility. Hum Reprod Update 10:39-51.

Said TM, Grunewald S, Paasch U, Glander HJ, Baumann T, Kriegel C, Li L, Agarwal A. 2005. Advantage of combining magnetic cell separation with sperm preparation techniques. Reprod Biomed Online 10:740-746.

Said T, Agarwal A, Grunewald S, Rasch M, Baumann T, Kriegel C, Li L, Glander HJ, Thomas AJ Jr, Paasch U. 2006. Selection of nonapoptotic spermatozoa as a new tool for enhancing assisted reproduction outcomes: An in vitro model. Biol Reprod 74:530-537.

Said TM, Agarwal A, Zborowski M, Grunewald S, Glander HJ, Paasch U. 2008. Utility of magnetic cell separation as a molecular sperm preparation technique. J Androl 29:134-142.

Sakkas D, Mariethoz E, St John JC. 1999. Abnormal sperm parameters in humans are indicative of an abortive apoptotic mechanism linked to the Fas-mediated pathway. Exp Cell Res 251:350-355.

Sakkas D, Moffatt O, Manicardi GC, Mariethoz E, Tarozzi N, Bizzaro D. 2002. Nature of DNA damage in ejaculated human spermatozoa and the possible involvement of apoptosis. Biol Reprod 66:1061-1067.

Sati L, Ovari L, Bennett D, Simon SD, Demir R, Huszar G. 2008. Double probing of human spermatozoa for persistent histones, surplus cytoplasm, apoptosis and DNA fragmentation. Reprod Biomed Online 16:570-579.

Sharlip ID, Jarow JP, Belker AM, Lipshultz LI, Sigman M, Thomas AJ, Schlegel PN, Howards SS, Nehra A, Damewood MD, et al. 2002. Best practice policies for male infertility. Fertil Steril 77:873882 .
Shen HM, Dai J, Chia SE, Lim A, Ong CN. 2002. Detection of apoptotic alterations in sperm in subfertile patients and their correlations with sperm quality. Hum Reprod 17:1266-1273.

Siddighi S, Patton WC, Jacobson JD, King A, Chan PJ. 2004. Correlation of sperm parameters with apoptosis assessed by dual fluorescence DNA integrity assay. Arch Androl 50:311-314.

Smolewski P, Bedner E, Du L, Hsieh TC, Wu JM, Phelps DJ, Darzynkiewicz Z. 2001. Detection of caspases activation by fluorochromelabeled inhibitors: Multiparameter analysis by laser scanning cytometry. Cytometry 44:73-82.

Taylor SL, Weng SL, Fox P, Duran EH, Morshedi MS, Oehninger S Beebe SJ. 2004. Somatic cell apoptosis markers and pathways in human ejaculated sperm: Potential utility as indicators of sperm quality. Mol Hum Reprod 10:825-834.

Tesarik J, Martinez F, Rienzi L, Iacobelli M, Ubaldi F, Mendoza C, Greco E. 2002. In-vitro effects of FSH and testosterone withdrawal on caspase activation and DNA fragmentation in different cell types of human seminiferous epithelium. Hum Reprod 17:1811-1819.

Thornberry NA, Lazebnik Y. 1998. Caspases: Enemies within. Science 281:1312-1316.

Thornberry NA, Bull HG, Calaycay JR, Chapman KT, Howard AD, Kostura MJ, Miller DK, Molineaux SM, Weidner JR, Aunins J. 1992. A novel heterodimeric cysteine protease is required for interleukin-1 beta processing in monocytes. Nature 356:768-774.

Vaux DL, Korsmeyer SJ. 1999. Cell death in development. Cell 96:245-254.

Wang X, Sharma RK, Sikka SC, Thomas AJ Jr, Falcone T, Agarwal A. 2003. Oxidative stress is associated with increased apoptosis leading to spermatozoa DNA damage in patients with male factor infertility. Fertil Steril 80:531-535.

Weng SL, Taylor SL, Morshedi M, Schuffner A, Duran EH, Beebe S, Oehninger S. 2002. Caspase activity and apoptotic markers in ejaculated human sperm. Mol Hum Reprod 8:984-991.

World Health Organization. 1999. Laboratory manual for the examination of human semen and sperm-Cervical mucus interaction,4th ed. Cambridge: Cambridge University Press.

Wuendrich K, Paasch U, Leicht M, Glander HJ. 2006. Activation of caspases in human spermatozoa during cryopreservation-An immonoblot study. Cell Tissue Bank 7:81-90.

Zheng S, Turner TT, Lysiak JJ. 2006. Caspase 2 activity contributes to the initial wave of germ cell apoptosis during the first round of spermatogenesis. Biol Reprod 74:1026-1033.

Zini A, Boman JM, Belzile E, Ciampi A. 2008. Sperm DNA damage is associated with an increased risk of pregnancy loss after IVF and ICSI: Systematic review and meta-analysis. Hum Reprod 23:26632668. 